\title{
Physical stability of solid dispersions with respect to thermodynamic solubility of tadalafil in PVP-VA
}

K.Wlodarski ${ }^{\mathrm{a}}$, W.Sawicki ${ }^{\mathrm{a}}$, A.Kozyra ${ }^{\mathrm{b}}$, L.Tajber ${ }^{\mathrm{b}}$

${ }^{a}$ Medical University of Gdansk, Department of Physical Chemistry, Hallera 107, 80-416 Gdansk, Poland

${ }^{\mathrm{b}}$ Trinity College Dublin, School of Pharmacy and Pharmaceutical Sciences, College Green,

Dublin 2, Ireland 


\section{Abstract}

The aim of this paper was to evaluate physical stability of solid dispersions in respect to the drug, tadalafil (Td), in vinylpyrrolidone and vinyl acetate block copolymer (PVP-VA). Nine solid dispersions of Td in PVP-VA (Td/PVP-VA) varied in terms of quantitative composition (1:9-9:1, w/w) were successfully produced by spray-drying. Their amorphous nature, supersaturated character and molecular level of mixing (a solid solution structure) were subsequently confirmed using DSC, PXRD, SEM and calculation of Hansen total solubility parameters. Due to thermal degradation of both components before the melting point of $\mathrm{Td}$ $\left(302.3{ }^{\circ} \mathrm{C}\right)$, an approach based on the drug crystallization from the supersaturated solid dispersion was selected to calculate the solubility of $\mathrm{Td}$ in the polymer. Annealing of the Td/PVP-VA solid dispersion $(1: 1, \mathrm{w} / \mathrm{w})$ at selected temperatures above its $T_{g}$ resulted in different stable solid dispersions. According to the Gordon-Taylor equation their new $T_{g} \mathrm{~s}$ gave the information about the quantitative composition which corresponded to the thermodynamic solubility of Td in PVP-VA at given temperatures of annealing. The obtained relationship was fitted to the exponential function, with the calculated solubility of $\mathrm{Td}$ of $20.5 \%$ at $25{ }^{\circ} \mathrm{C}$. This value was in accordance with the results of hot stage polarizing light microscopy as well as stability tests carried out at $80^{\circ} \mathrm{C}$ and $0 \% \mathrm{RH}$, in which Td solid dispersions containing 10$20 \%$ of the drug were the only systems that did not crystallize within two months. A thermal analysis protocol utilizing a fast heating rate was shown to generate $\mathrm{Td}$ solubility data complementing the solid dispersion method. The Flory-Huggins model applied for the Td/PVP-VA system yielded the solubility value of $0.1 \%$ at $25{ }^{\circ} \mathrm{C}$, showing the lack of applicability in this case.

Keywords: Amorphous solid dispersion, Tadalafil, Polymer, Physical stability, Crystallization, Thermodynamic solubility

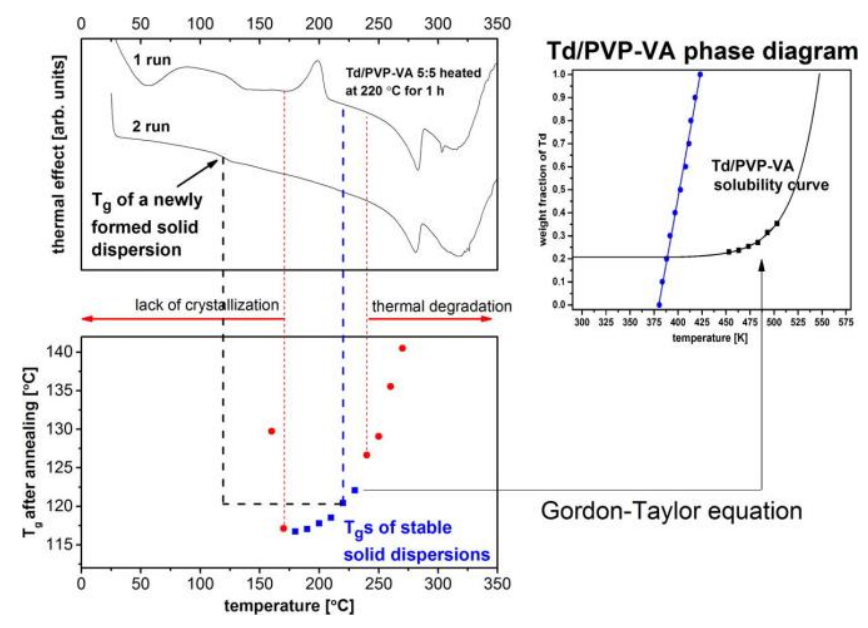




\section{Introduction}

The number of poorly soluble drug substances available on the global pharmaceutical market has been growing constantly for last decades. Hence, many approaches aimed at improving apparent solubility and increasing dissolution rates have been developed [5,21]. Formation of solid dispersions is one of the most promising approaches due to its high effectiveness, flexibility in designing the composition and up-to-date devices adapted for their production $[4,20]$. In general, solid dispersions can be defined as at least two-component systems of a drug substance usually dispersed in a polymeric matrix but in fact they may be more diverse in their composition [11]. The unique morphology of solid dispersions frequently leads to water solubility enhancement, which can be explained by improved wettability, amorphization and size reduction of drug particles [21].

Despite many advantages and the fact that solid dispersions have been known for the last 40 years, only a few of them have been introduced as commercial products to date [3]. The main reason of this lies in their physical instability, which may lead to changes in dissolution profiles and, as a consequence, in bioavailability [8]. Solid dispersions are preferably amorphous systems where the drug substance is molecularly dispersed in an inherently amorphous polymer. The initial drug loading may be boundlessly high but from the thermodynamic point of view only the amount not exceeding its intrinsic solubility in a polymer does not tend to crystallize [13]. This gives an advantage over amorphous drugs without excipients, which are always high energy and therefore highly physically unstable structures. Crystallization can be considered as a kinetically driven process depending on mobility of molecules but thermodynamic properties resulting from the drug content and its solubility in the polymer are thought to be the key parameters regarding physical stability of solid dispersions $[13,15]$.

Considering the above, the aim of this paper was to evaluate the physical stability of supersaturated and undersaturated solid dispersions in respect to the drug, tadalafil (Td), in vinylpyrrolidone and vinyl acetate block copolymer (PVP-VA). The drug and polymer were selected based on our previous study in which aqueous solubility and the dissolution rate of Td were significantly improved by its incorporation in the PVP-VA matrix [23]. An interesting aspect of the current work is that the glass transition temperature $\left(T_{g}\right)$ of the polymer $\left(107.4{ }^{\circ} \mathrm{C}\right)$ is lower than that of pure amorphous $\mathrm{Td}\left(149.9^{\circ} \mathrm{C}\right)$. Since it is deemed that the stabilizing effect on the amorphous drug is mainly the result of its $T_{g}$ increase and molecular mobility 
decrease in a solid dispersion $[9,10]$, investigating properties of a system with the reverse trend in $T_{g}$ s may contribute to a better understanding of stabilization mechanisms.

Based on the previous data [22] on the thermal degradation of Td and PVP-VA before reaching the melting point of the drug it was paramount to select an appropriate method to determine thermodynamic solubility of Td in PVP-VA at $25^{\circ} \mathrm{C}$, which is the most common temperature of drug storage. It is important as to date all described methods of solubility determination are based on measurements at temperatures above $T_{g}$ of the polymer and one of the most common protocols known as "the melting point depression method" utilizes the decrease of the drug melting temperature in a physical mixture [13]. Apart from thermal degradation, the biggest drawback of this method is the risk of solubility underestimation caused by low molecular mobility in a viscous polymer and an inadequate shift of the endotherm. Modifications involving initial milling of physical mixtures, annealing and different heating rates improve the protocol but significantly extend the analysis $[18,17]$.

Another, a slightly modified approach based on the melting point depression principle, which also could not be utilized in this work, is based on the measurement of the drug melting enthalpy reduction in a physical mixture [1]. In this method a crystalline drug dissolves in a polymer at a pre-set constant temperature and is subsequently heated up to the drug melting point to determine its enthalpy of fusion. The analysis of physical mixtures prepared in different weight ratios enables to extrapolate the enthalpy curve to "the zero melting enthalpy" point which indicates the complete dissolution of a drug in a polymer and thereby the thermodynamic solubility at a given temperature of dissolving. Finally, by repeating this step at different dissolving temperatures one can obtain the solubility change as a function of temperature.

Thus, to determine solubility of $\mathrm{Td}$ in PVP-VA considering thermal degradation of components, an innovative protocol described by Mahieu and coworkers was selected [12]. The general principle of this approach employs drug crystallization from a supersaturated solid dispersion, which takes place above its $T_{g}$. As a consequence, a thermodynamically stable solid dispersion is formed, of which $T_{g}$ gives the information about its new quantitative composition. To support this approach, another method of drug solubility in the polymer, based on a fast heating rate, was investigated. This experimental protocol utilized an annealing step whereby physical mixtures of crystalline Td and PVP-VA were equilibrated at a chosen temperature above the $T_{g}$ of the polymer and subsequently the melting point of the drug was determined applying a heating rate of $200{ }^{\circ} \mathrm{C} / \mathrm{min}$. It has been established that thermal decomposition of 
pharmaceutical substances can be minimized when using sufficiently fast heating rates [14]. The duration of the annealing step was optimized to allow the drug to dissolve in the amorphous polymer, similar to the treatment used by Sun et al. [17]. Solid dispersions of Td in PVP-VA (Td/PVP-VA) in this investigation were produced by spray-drying. Finally, they were subjected to accelerated aging tests, the results of which were juxtaposed with the calculated solubility of Td in PVP-VA and hot stage polarized light microscopy experiments.

2. Materials and methods

\subsection{Materials}

Tadalafil (Td) (series 20211) as well as vinylpyrrolidone and vinyl acetate block copolymer (Kollidon VA 64, PVP-VA) were kindly donated by Polpharma S.A. (Poland) and BASF SE (Germany), respectively. Ultrapure water was produced by Millipore Direct-Q 3UV-R water purification system. Acetone and all other chemicals of analytical grade were purchased from Sigma-Aldrich (Germany).

\subsection{Methods}

\subsubsection{Preparation of samples}

\subsubsection{Preparation of tadalafil solid dispersions using spray drying}

Td/PVP-VA solid dispersions were obtained by spray drying of solutions (acetone/water 9:1, $\mathrm{v} / \mathrm{v})$ containing Td and PVP-VA in a range of weight ratios (1:9-9:1 w/w in 10\% increments). The total concentration of solids in solutions was $1 \%(\mathrm{w} / \mathrm{v})$. The process was performed in a Mini Spray Dryer B-290 (Büchi, Switzerland) using an open, suction mode with nitrogen. The gas inlet pressure was 6 bar and set to $4 \mathrm{~cm}$ of the gas flow (rotameter setting). Spray dryer was equipped with a standard atomization nozzle with a $1.5-\mathrm{mm}$ cap and a $0.7-\mathrm{mm}$ tip. The pump speed was set to $25 \%(7 \mathrm{ml} / \mathrm{min})$ and the aspirator was operated at $100 \%$. The inlet temperature was set to $65{ }^{\circ} \mathrm{C}$ and such setup resulted in an outlet temperature of $52{ }^{\circ} \mathrm{C}$. Additionally, $\mathrm{Td}$ $1 \%(\mathrm{w} / \mathrm{v})$ solution (acetone/water 9:1, v/v) was spray dried following the above procedure.

\subsubsection{Preparation of tadalafil physical mixtures}

The equivalent physical mixtures (comprising PVP-VA and crystalline $\mathrm{Td}$ in the same proportions as those used for spray drying) were prepared by milling in a planetary ball mill PM 100 (Retsch, Germany) at 400 RPM for $10 \mathrm{~min}$. A quantity of $2 \mathrm{~g}$ of powder was loaded 
to the stainless steel container, which had a volume of $50 \mathrm{ml}$, and three stainless steel balls (20 $\mathrm{mm}$ in diameter, $32 \mathrm{~g}$ each) were used for all milling experiments.

\subsubsection{Calculation of thermodynamic solubility of tadalafil in PVP-VA}

2.2.2.1. Conventional differential scanning calorimetry (DSC)

Thermodynamic solubility of Td in PVP-VA was investigated calorimetrically using a DSC 821e STAR $^{\mathrm{e}}$ system (Mettler-Toledo, Switzerland) controlled by Mettler Toledo STAR ${ }^{\mathrm{e}}$ software. To determine the relationship between the content of $\mathrm{Td}$ and a glass transition temperature $\left(T_{g}\right)$ of the solid dispersions, binary spray dried samples as well as pure PVP-VA and spray dried Td were subjected to calorimetric measurements in $40 \mu \mathrm{l}$ aluminum pans at a heating rate of $10{ }^{\circ} \mathrm{C} / \mathrm{min}$ in the temperature range of $25-350{ }^{\circ} \mathrm{C}$ under nitrogen atmosphere $(60 \mathrm{ml} / \mathrm{min})$.

To calculate the thermodynamic solubility of Td in PVP-VA, the Td/PVP-VA solid dispersion $(5: 5, \mathrm{w} / \mathrm{w})$ was heated $\left(10^{\circ} \mathrm{C} / \mathrm{min}\right)$ and annealed at six different temperatures ranging from 180 to $230{ }^{\circ} \mathrm{C}$ for one hour. Samples were then cooled to ambient temperature and subjected to the previously described heating method $\left(25-350{ }^{\circ} \mathrm{C}, 10{ }^{\circ} \mathrm{C} / \mathrm{min}\right)$ to determine $T_{g} \mathrm{~s}$ of the newly formed solid dispersions. In all calorimetric experiments $T_{g} \mathrm{~s}$ were determined as midpoints of the heat flow change, while for exo- and endothermic events the onset temperatures were evaluated. All experiments were performed in triplicate followed by calculation of average values.

\subsubsection{Melting point depression measurements - High Speed DSC (HSDSC)}

The melting events of Td in the physical mixtures prepared as described in Section 2.2.1.2 were measured using a PerkinElmer Diamond DSC unit (Waltham, MA, USA) with HyperDSC. The unit was refrigerated using an ULSP B.V. 130 cooling system (Ede, Netherlands). The gas flow was controlled at $40 \mathrm{~mL} / \mathrm{min}$ using a PerkinElmer Thermal Analysis Gas Station (TAGS). The instrument was calibrated for both melting onset and enthalpy with indium at $200{ }^{\circ} \mathrm{C} / \mathrm{min}$. Before the measurement, samples in the DSC standard aluminum pans $(\sim 1 \mathrm{mg})$ were first annealed in an oven (Memmert, Germany) at $120{ }^{\circ} \mathrm{C}$ for $2 \mathrm{~h}$. The annealing time was chosen to be $2 \mathrm{~h}$ based on comparison of the heat fusion values obtained for the Td and PVP-VA (9:1, w/w) physical mixture, non-annealed and annealed for 2, 4, and $6 \mathrm{~h}$. Samples were then cooled down to room temperature and the final sample weight was calculated. The samples were 
heated from $0{ }^{\circ} \mathrm{C}$ to $340{ }^{\circ} \mathrm{C}$ at a heating rate of $200{ }^{\circ} \mathrm{C} / \mathrm{min}$ in the DSC instrument and melting points recorded. The heating rate of $200{ }^{\circ} \mathrm{C} / \mathrm{min}$ was the most suitable for the Td/PVP-VA solid dispersion. It was chosen based on comparison of the thermal events of pure Td and pure PVPVA obtained when $50{ }^{\circ} \mathrm{C} / \mathrm{min}, 100{ }^{\circ} \mathrm{C} / \mathrm{min}$ and $200{ }^{\circ} \mathrm{C} / \mathrm{min}$ heating rates were applied.

\subsubsection{Hansen solubility parameters}

Hansen total solubility parameter $\left(\delta_{t}\right)$ of Td and PVP-VA was calculated based on the group contribution method developed by van Krevelen and co-workers [19]. This approach utilizes the molecular density of energies coming from dispersion $\left(\delta_{d}\right)$, dipolar $\left(\delta_{p}\right)$ and hydrogen forces $\left(\delta_{h}\right)$ according to Eq. (1):

$$
\delta_{t}^{2}=\delta_{d}^{2}+\delta_{p}^{2}+\delta_{h}^{2}
$$

A small difference in total solubility parameters between two compounds $\left(\Delta \delta_{t}<7 \mathrm{MPa}^{0.5}\right)$ indicates that their miscibility is likely to occur [6].

\subsubsection{Physicochemical analysis of tadalafil solid dispersions}

\subsubsection{Powder X-ray diffraction (PXRD) analysis}

X-ray analysis of samples was made using a desktop powder diffractometer MiniFlexII (Rigaku, Japan), equipped with a $\mathrm{Cu} \mathrm{K \alpha}$ radiation $\mathrm{X}$-ray source operated under a voltage of $30 \mathrm{kV}$ and current of $15 \mathrm{~mA}$. The samples were placed on a low-background silicon holder and scanned at room temperature over a $2 \theta$ range of $5-40^{\circ}$ at a step size of $0.05^{\circ}$ s.

\subsubsection{Thermogravimetric analysis (TGA)}

Thermal stability of Td and PVP-VA under heating conditions used in DSC analysis (Section 2.2.2.1) was investigated by Mettler TG 50 thermogravimetric analyzer (Mettler-Toledo, Switzerland) linked to a Mettler MT5 balance (Mettler-Toledo, Switzerland). Samples in open aluminum pans were placed in a furnace under nitrogen purge $(30 \mathrm{ml} / \mathrm{min})$, heated $\left(10^{\circ} \mathrm{C} / \mathrm{min}\right)$ and annealed for one hour at different temperatures ranging from $210{ }^{\circ} \mathrm{C}$ to $270{ }^{\circ} \mathrm{C}$. Degradation of Td and PVP-VA was determined by the weight loss percentage of the sample. 


\subsubsection{True density of powders}

The true density of PVP-VA and spray dried Td was measured by an AccuPyc 1330 Pycnometer (Micromeritics®) using helium to determine the volume of the sample. A $1 \mathrm{~cm}^{3}$ sample cup was used.

\subsubsection{Scanning electron microscopy (SEM) analysis}

Morphological assessment of Td solid dispersions in PVP-VA (5:5, w/w) was carried out on a Supra variable Pressure Field Emission Scanning Electron Microscope (Zeiss, Germany) equipped with a secondary electron detector and accelerating voltage of $5 \mathrm{kV}$. A small amount of powder was placed directly on the aluminum stub covered with a carbon tab and excess of powder was removed using a nitrogen purge. The samples were sputter coated with gold/palladium under vacuum before analysis.

\subsubsection{Hot stage optical and polarized light microscopy (HS PLM)}

Optical and polarized light microscopy was performed with an Olympus BX53 polarizing optical microscope equipped with a U-POT cross polarizer, a Q IMAGING Fast 1394 camera and a Linkam LTS 420 hotstage. Samples were spread evenly on a glass slide and heated at a $5{ }^{\circ} \mathrm{C} / \mathrm{min}$ rate.

\subsubsection{Accelerated aging tests}

To examine physical stability, all solid dispersions and neat amorphous Td were subjected to the following aging conditions: $25^{\circ} \mathrm{C}$ and $75 \% \mathrm{RH} ; 40{ }^{\circ} \mathrm{C}$ and $0 \% \mathrm{RH} ; 40{ }^{\circ} \mathrm{C}$ and $75 \% \mathrm{RH}$; $80{ }^{\circ} \mathrm{C}$ and $0 \% \mathrm{RH}$ and $130{ }^{\circ} \mathrm{C}$ and $0 \% \mathrm{RH}$. The presence of crystalline Td was investigated after pre-set time intervals using PXRD.

\section{Results and discussion}

3.1. Calculation of tadalafil thermodynamic solubility in a polymer

\subsubsection{Physicochemical analysis of tadalafil solid dispersions}

Utilization of solid dispersions instead of physical mixtures in the evaluation of drug-polymer solubility is one of the features that distinguishes this method from other commonly used, based on melting point depression [18,17]. However, the applicability of this approach depends 
strongly on the type of a solid dispersion which must reveal an amorphous and supersaturated character and good miscibility between components at the molecular level.

In this work a range of Td/PVP-VA solid dispersions with nine different weight ratios was successfully obtained by spray-drying, in contrast to Mahieu and coworkers who used ball milling [12]. The same drying conditions were also used for Td solution without excipients. As indicated by the disappearance of Bragg peaks characteristic of crystalline Td, PXRD revealed the amorphous nature of all solid dispersions and spray dried Td (Fig. 1). What is crucial for further analysis, inherently amorphous PVP-VA did not mask peaks of crystalline Td in any of the corresponding physical mixtures and the X-ray diffraction pattern of the sample containing the least amount of the drug $(1: 9$, w/w) is presented in Fig. 1.

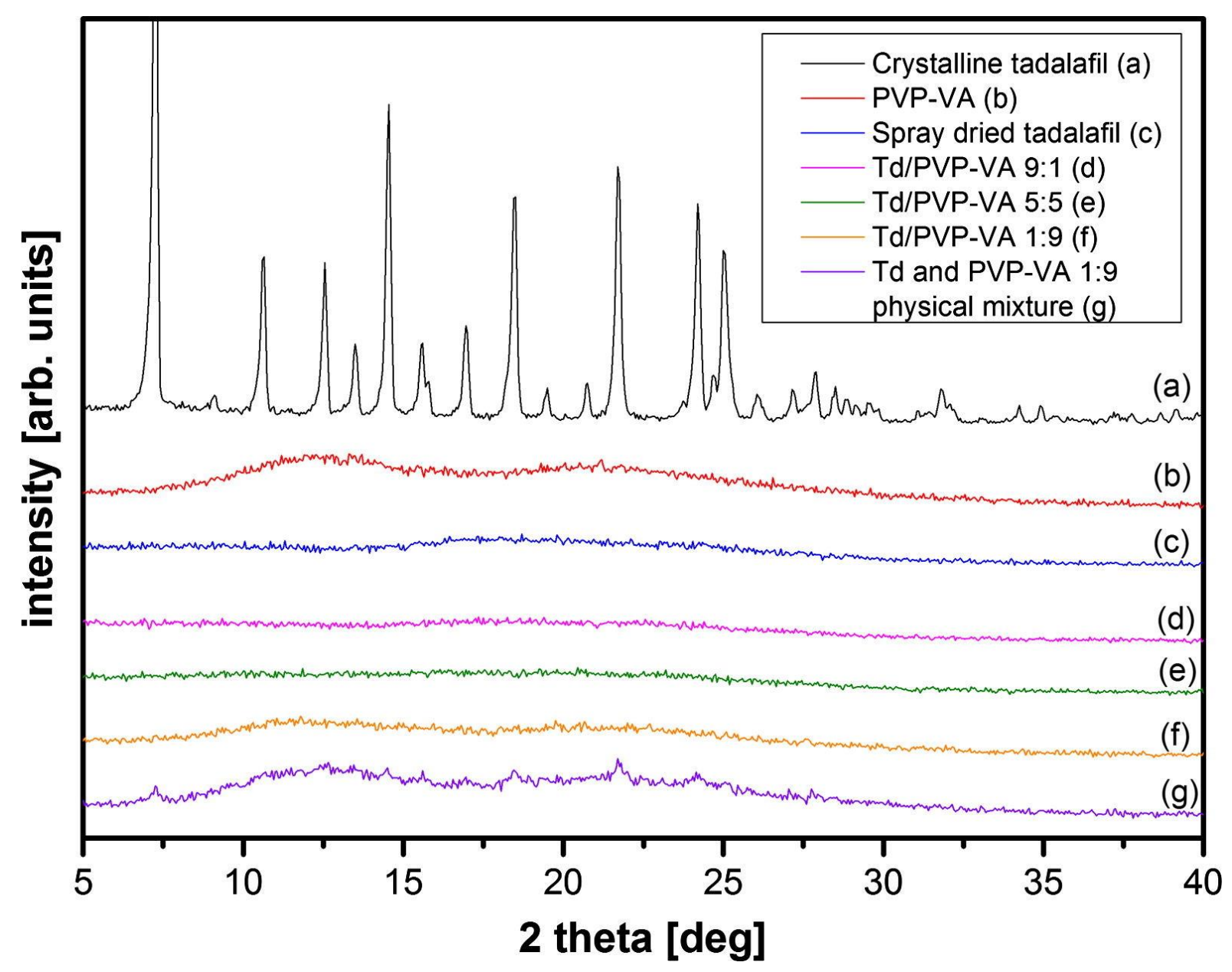

Fig. 1. X-ray powder diffraction patterns of crystalline tadalafil (Td) (a), PVP-VA (b), spray dried tadalafil (c), 9:1 (w/w) Td/PVP-VA solid dispersion (d), 5:5 (w/w) Td/PVP-VA solid dispersion (e), 1:9 (w/w) Td/PVP-VA solid dispersion (f), 1:9 (w/w) Td and PVP-VA physical mixture (g). 
The lack of Bragg peaks was not the result of the particle size reduction to the order of nanometers which might result in a decrease in the intensity of peaks. As depicted in Fig. 2, the particles were typical of spray dried material and were round-shaped and micrometer-sized.
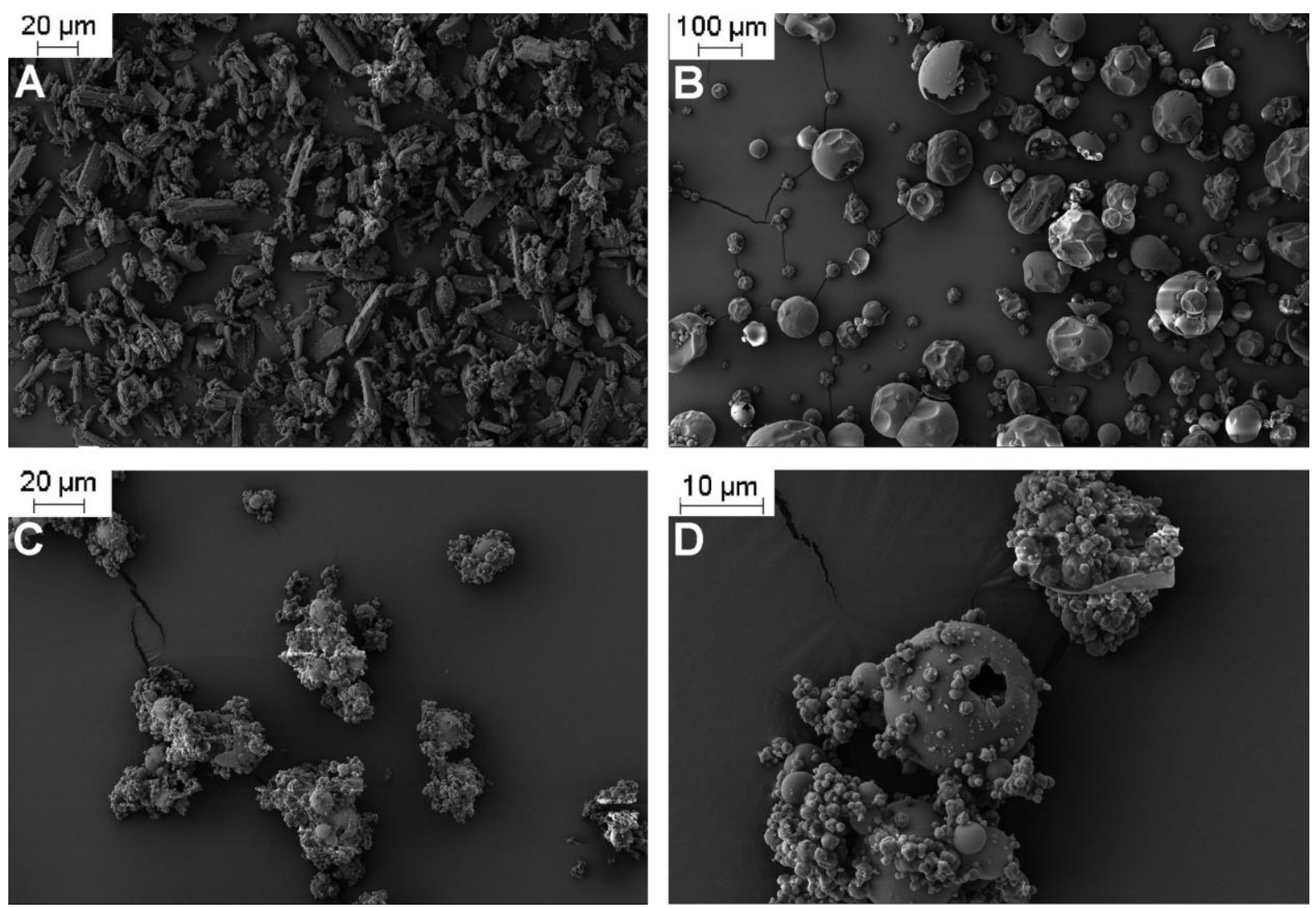

Fig. 2. SEM images of crystalline tadalafil (Td) (A), PVP-VA (B), 5:5 (w/w) Td/PVP-VA solid dispersion $(C, D)$.

For a more complete assessment of the physicochemical nature of solid dispersions, the samples were subjected to thermal analysis $\left(25-350{ }^{\circ} \mathrm{C}, 10^{\circ} \mathrm{C} / \mathrm{min}\right)$, which confirmed the chaotic arrangement of the molecules in solid dispersions as well as in spray dried Td (Figs. 3a and $3 \mathrm{~b}$ ). In the thermogram of the latter system there is a $T_{g}$ with a midpoint at $149.9{ }^{\circ} \mathrm{C}$, followed by an exothermic event with an onset at $181.6^{\circ} \mathrm{C}$ and an endothermic sharp peak at $302.1{ }^{\circ} \mathrm{C}$. Melting of crystalline $\mathrm{Td}$, which starts at $302.3{ }^{\circ} \mathrm{C}$, indicates that the exotherm refers to sample crystallization. The occurrence of crystallization during heating of the amorphous sample places Td in the group II of the glass-forming drugs [2]. It is a requirement for the successful application of the current solubility method that a solid dispersion undergoes crystallization when annealed at a pre-set temperature. Drugs falling into group I, showing crystallization during cooling from the liquid (molten) state prior to the $T_{g}$ transition, may 
perhaps also be suitable candidates when stabilized in solid dispersions, while solubility of drugs not crystallizing during reheating (group III), cannot be assessed using the annealing approach.

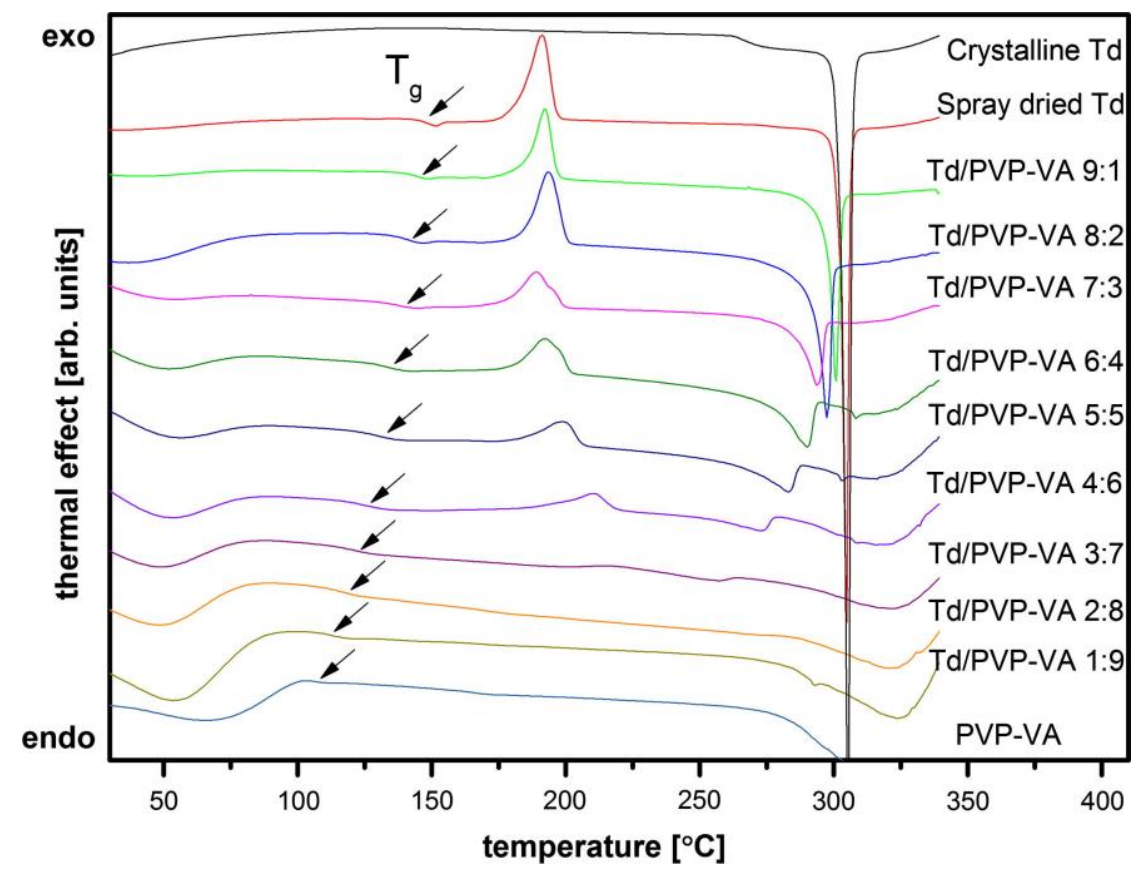

Fig. 3a. DSC thermograms of (from top to bottom) crystalline tadalafil (Td), spray dried tadalafil, 9:1-1:9 (w/w) Td/PVP-VA solid dispersions, PVP-VA obtained at a heating rate of $10{ }^{\circ} \mathrm{C} / \mathrm{min}$. Arrows indicate $T_{g} \mathrm{~s}$ of the systems.

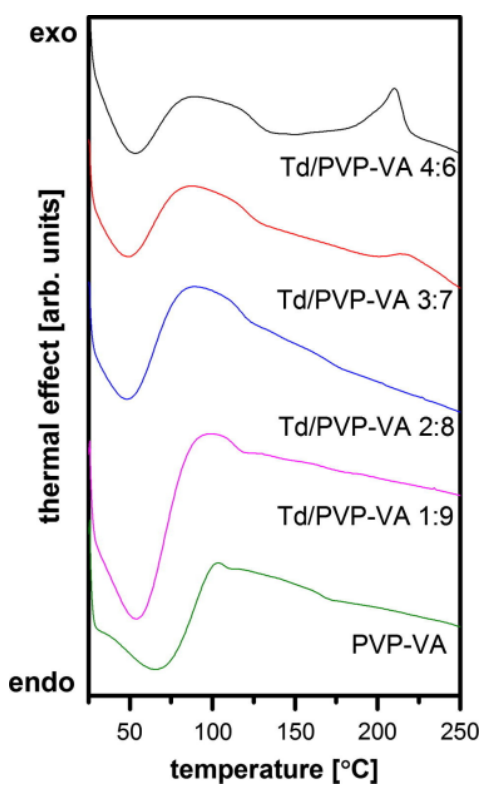

Fig. 3b. $T_{g} \mathrm{~s}$ of (from top to bottom) 4:6-1:9 Td/PVP-VA solid dispersions and PVP-VA obtained at a heating rate of $10^{\circ} \mathrm{C} / \mathrm{min}$. 
Thermal analysis of the Td/PVP-VA solid dispersions revealed only one $T_{g}$ per each system, occurring between the $T_{g}$ points of pure PVP-VA $\left(107.4{ }^{\circ} \mathrm{C}\right)$ and amorphous $\mathrm{Td}\left(149.9^{\circ} \mathrm{C}\right)$, as presented in Figs. $3 \mathrm{a}$ and $3 \mathrm{~b}$. The detailed analysis of the $T_{g}$ dependence on the composition of a solid dispersion is placed in Section 3.1.2. A single $T_{g}$ for all solid dispersions with 10-90\% of $\mathrm{Td}$ indicates good miscibility of both components and the formation of molecular level amorphous solid dispersions, that is solid solutions.

Despite a decrease in $T_{g} \mathrm{~s}$ of solid dispersions in comparison with spray dried Td, crystallization of the drug starts above $180{ }^{\circ} \mathrm{C}$, similar to the amorphous form of $\mathrm{Td}$ without the polymer. Nevertheless, crystallization is closely related to the quantitative composition of a solid dispersion. Due to certain solubility of Td in PVP-VA the enthalpy of crystallization decreases when the amount of the polymer increases. The lack of exotherms in solid dispersions with $\mathrm{Td}$ content of less than $30 \%$ may indicate the complete dissolution at $180{ }^{\circ} \mathrm{C}$ but, on the other hand, might be the result of a small amount of $\mathrm{Td}$ in samples and therefore lack of crystallization detection (Fig. 3b). It is however certain that if crystallization occurs in a solid dispersion, it indicates its supersaturated nature in respect to the drug at a temperature this process takes place.

The endothermic sharp peak observed for crystalline and amorphous $\mathrm{Td}$ above $302{ }^{\circ} \mathrm{C}$ and defined for both substances as melting is shifted to lower temperatures for solid dispersions. The reason of this is dissolving of previously crystallized $\mathrm{Td}$ in a polymeric matrix as a result of its increased solubility at higher temperatures. The more polymer in a solid dispersion the more shifted and blurred dissolution endotherm, which can be easily distinguished from the melting phenomenon. In the case of solid dispersions showing no crystallization, this dissolution process does not occur and thereby another endothermic event related to thermal degradation of the polymer can be seen (Fig. 3a).

Thermal degradation of PVP-VA can be observed as the pronounced and distorted endothermic event occurring above $275^{\circ} \mathrm{C}$ for the solid dispersions with the highest content of the polymer and pure polymer. Furthermore, the thermogram of crystalline Td likewise indicates its degradation above $260{ }^{\circ} \mathrm{C}$, visible as slight lowering of the baseline (Fig. 3a), which is confirmed by the change of powder's color from white to brown and release of smoke. Thermogravimetric analysis carried out for both components showed $2.0 \%$ and $11.3 \%$ weight loss for Td and PVP-VA at $302{ }^{\circ} \mathrm{C}$, respectively (Fig. 4). Thus, thermal degradation of Td and PVP-VA, even at a heating rate of $10{ }^{\circ} \mathrm{C} / \mathrm{min}$, prevents the use of conventional methods for 
solubility assessment, which requires reliable measurements of the melting events of the drug $[13,18,17]$.

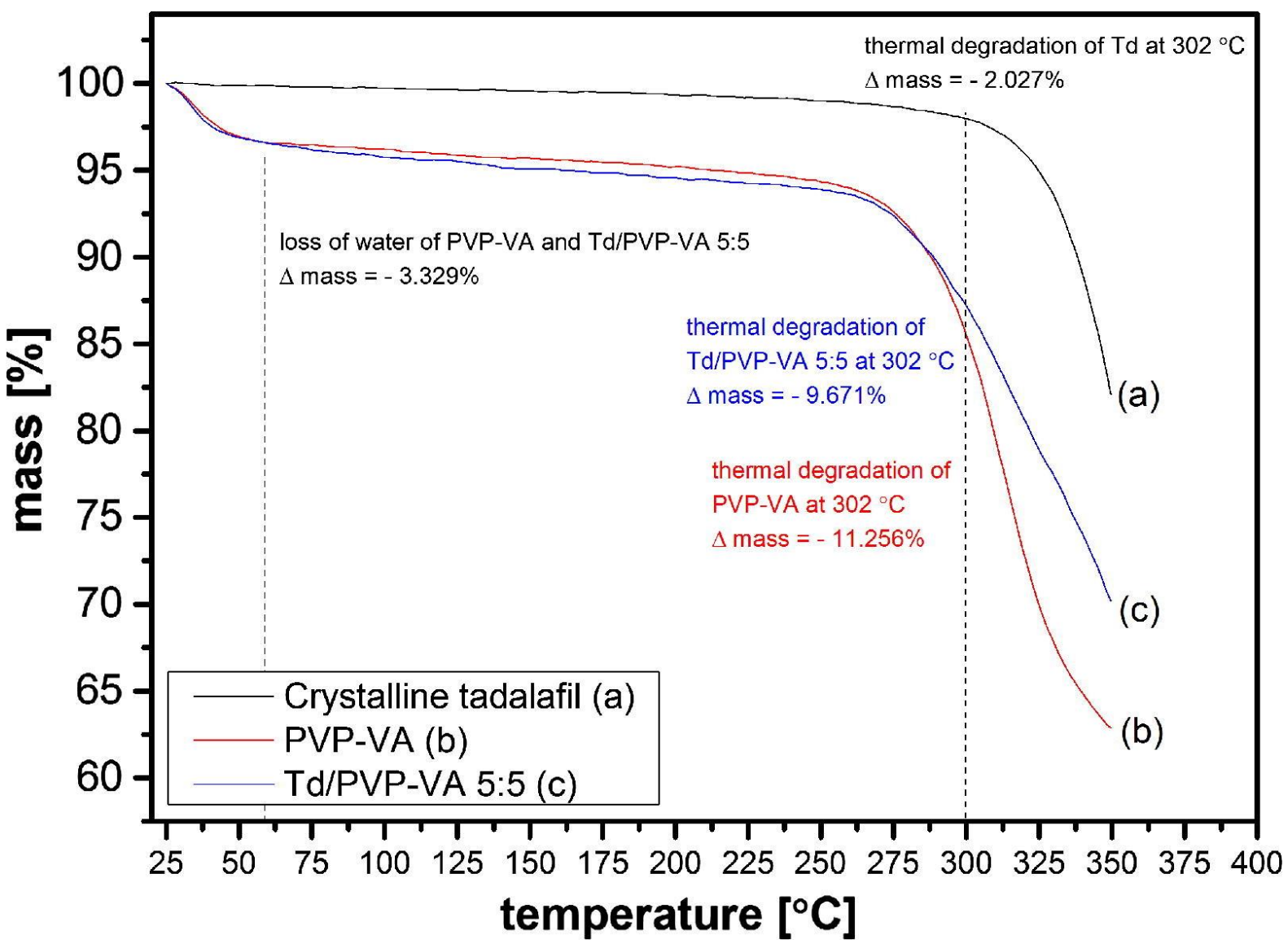

Fig. 4. Thermogravimetric curves illustrating thermal decomposition of crystalline tadalafil (Td) (a), PVP-VA (b), 5:5 (w/w) Td/PVP-VA solid dispersion (c) at a heating rate of $10{ }^{\circ} \mathrm{C} / \mathrm{min}$

\subsubsection{The $\mathrm{T}_{\mathrm{g}} \mathrm{s}$ dependence on the composition in tadalafil solid dispersions}

Crystallization of $\mathrm{Td}$ from a supersaturated solid dispersion leads to the formation of a thermodynamically stable solid dispersion. The measurement of its $T_{g}$ gives direct information on its composition if the relationship between $T_{g} \mathrm{~s}$ and the drug content in solid dispersions is previously shown. The Gordon-Taylor equation introduced in 1952 describes this dependence (Eq. (2)) [7]:

$$
T_{g(\mathrm{Td} / \mathrm{PVP}-\mathrm{VA})}=\frac{X_{(\mathrm{Td})} T_{g(\mathrm{Td})}+K X_{(\mathrm{PVP}-\mathrm{VA})} T_{g(\mathrm{PVP}-\mathrm{VA})}}{X_{(\mathrm{Td})}+K X_{(\mathrm{PVP}-\mathrm{VA})}}
$$


where $X_{(\mathrm{Td})}$ and $X_{(\mathrm{PVP}-\mathrm{VA})}$ are weight fractions of Td and PVP-VA in a solid dispersion, respectively. $K$ is a constant value which can be calculated based on the following Eq. (3)[16]:

$$
K=\frac{p_{(\mathrm{Td})} T_{g(\mathrm{Td})}}{p_{(\mathrm{PVP}-\mathrm{VA})} T_{g(\mathrm{PVP}-\mathrm{VA})}}
$$

where $p_{(\mathrm{Td})}$ and $\mathrm{p}_{(\mathrm{PVP}-\mathrm{VA})}$ are true densities of amorphous Td and PVP-VA, respectively. The $K$ value determines the shape of the function $T_{g}=f\left(X_{(\mathrm{Td})}\right)$, which assumes a linear dependence as $K$ is equal to 1 . For the Td and PVP-VA systems the $K$ value is 1.16 , which results in an almost linear relationship as presented in Fig. 5 (green triangles) and defined by the equation: $T_{g \text { (Td/PVP- }}$ VA) $=42.364 X_{(\mathrm{Td})}+106.51, R^{2}=0.9983$.

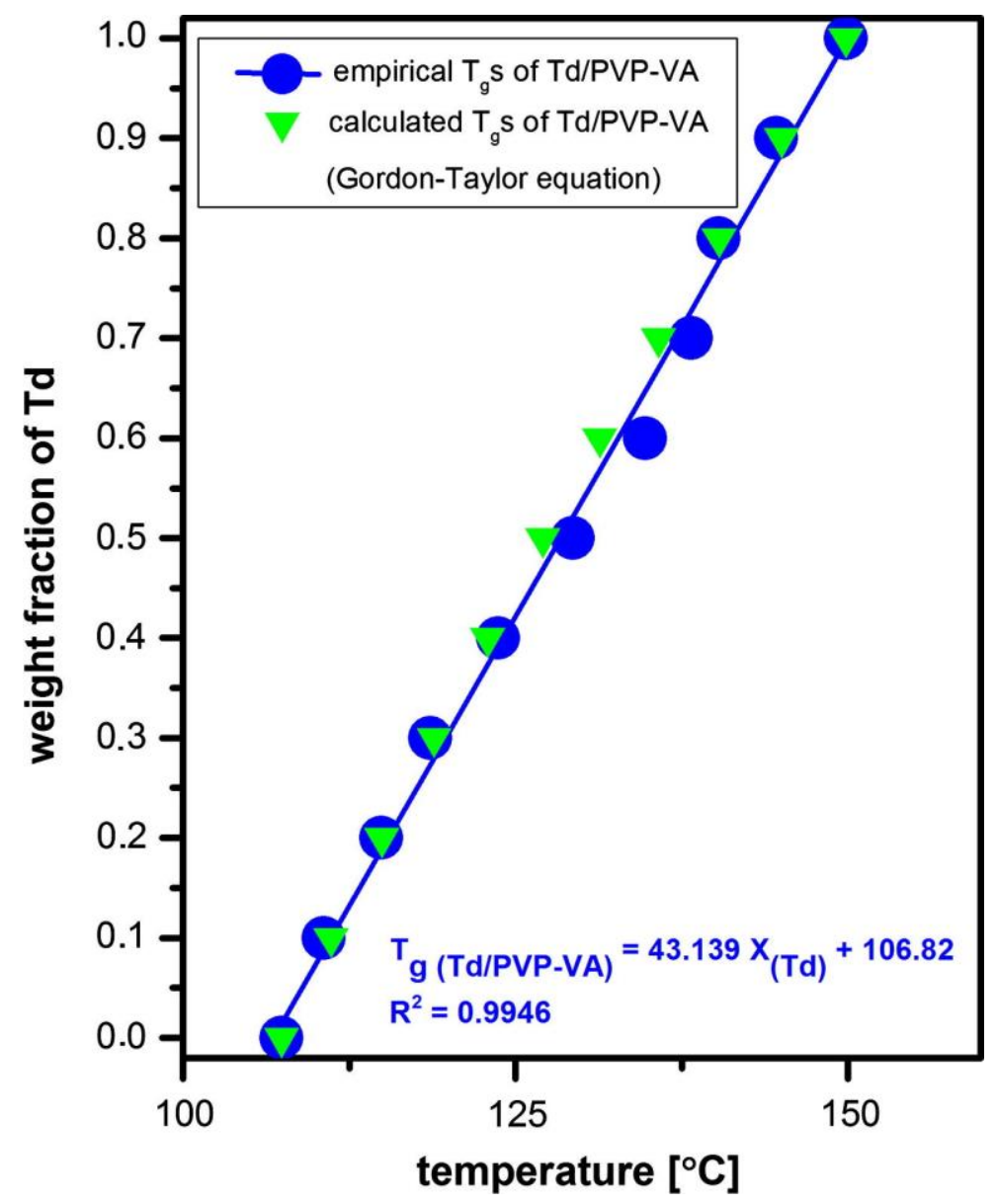

Fig. 5. The green triangles present the relationship between $T_{g} \mathrm{~s}$ and the composition of Td/PVP-VA solid dispersions which was calculated based on the Gordon-Taylor equation while the blue connected dots present the same relationship obtained empirically and described by the linear function $T_{g(\mathrm{Td} / \mathrm{PVP}-\mathrm{VA})}=43.139 X_{(\mathrm{Td})}+106.82, R^{2}=0.9946$. 
The linear dependence between $T_{g}$ and the content of Td was confirmed empirically, measuring the $T_{g}$ s of nine different Td/PVP-VA solid dispersions (Fig. 3a). The experimental relationship can be described by the formula: $T_{g(\mathrm{Td} / \mathrm{PVP}-\mathrm{VA})}=43.139 X_{(\mathrm{Td})}+106.82, R^{2}=0.9946$ and this equation was selected for further solubility calculations (Fig. 5). As the Gordon-Taylor equation can be used solely in the case of ideally mixed solid solutions, a good agreement with the calculated data indicates good molecular miscibility and lack of strong chemical interactions between Td and PVP-VA. The slight shifts of experimentally obtained $T_{g}$ s toward higher temperatures for Td/PVP-VA solid dispersions $(5: 5-7: 3$, w/w), when compared to calculated $T_{g} \mathrm{~s}$, may reveal possible hydrogen bonds between Td amino groups and PVP-VA carbonyl groups in this range of weight ratios (Fig. 5). Furthermore, good miscibility can be confirmed by a small difference in calculated Hansen total solubility parameters of $4.1 \mathrm{MPa} 0.5$ (25.7 $\mathrm{MPa}^{0.5}$ for Td and $21.6 \mathrm{MPa}^{0.5}$ for PVP-VA).

\subsubsection{Formation of tadalafil saturated solid dispersions}

Having determined the relationship between $T_{g} \mathrm{~s}$ and the composition of solid dispersions, the final protocol of solubility measurement was developed. Briefly, it assumes some crystallization of the excess of $\mathrm{Td}$ from the supersaturated solid dispersion and examination of $T_{g}$ s of the newly formed solid dispersion to determine its composition (Section 2.2.2.1). Annealing at different temperatures above the crystallization onset leads to a different degree of crystallization resulting from different solubility of Td in PVP-VA. Therefore, this enables solid dispersions which are thermodynamically stable at their annealing temperatures to be obtained.

Theoretically, each Td/PVP-VA solid dispersion exhibiting crystallization upon heating, which is that with Td content ranging from $40 \%$ to $90 \%$, could be selected for analysis. On the one hand, a high Td to PVP-VA weight ratio is desired to provide the broad solubility measurement spectrum but, from the practical point of view, the low amount of the polymer might result in a low quantity of a newly formed solid dispersion and thereby issues with $T_{g}$ detection. To illustrate this, by selecting the Td/PVP-VA solid dispersions $(9: 1, \mathrm{w} / \mathrm{w})$ one can determine the drug solubility ranging from $0 \%$ to $90 \%$ of $\mathrm{Td}$, but in the case of low solubility the amount of a newly formed solid dispersion might constitute only slightly more than $10 \%$ of the sample weight. Indeed, crystallization of Td from solid dispersions containing less than $50 \%$ of the polymer resulted in no visible $T_{g} \mathrm{~s}$ in the second heating run. Thus, the Td/PVP-VA solid dispersion $(5: 5, w / w)$ was selected for further analysis and the maximum solubility of Td in 
PVP-VA that could be determined was therefore 50\%. Notwithstanding, as it is shown later in Section 3.1.3, higher solubility of Td in PVP-VA occurs in the range of temperatures where thermal degradation of components prevents reliable examination.

Another crucial factor affecting the results of this study is the sample annealing time, which must be long enough to enable crystallization of the entire excess of Td from the Td/PVP-VA solid dispersion $(5: 5, \mathrm{w} / \mathrm{w})$. The time of annealing was optimized at the lowest accepted temperature of the process, which is at $180{ }^{\circ} \mathrm{C}$ (Fig. 6), as at any higher temperature the crystallization process proceeds faster due to a decrease in viscosity of the polymer. Half-hour, one-hour and two-hour annealing of the Td/PVP-VA solid dispersion (5:5, w/w) followed by cooling and reheating of the sample resulted in new $T_{g}$ values of $117.23 \pm 0.08{ }^{\circ} \mathrm{C}$, $116.73 \pm 0.14{ }^{\circ} \mathrm{C}$ and $116.81 \pm 0.10{ }^{\circ} \mathrm{C}$, respectively. The higher $T_{g}$ of a solid dispersion annealed for half-hour was the evidence of incomplete Td crystallization, while the other two $T_{g} \mathrm{~s}$ did not differ significantly. Thus, the annealing time of one-hour was selected as more favorable for thermolabile substances.

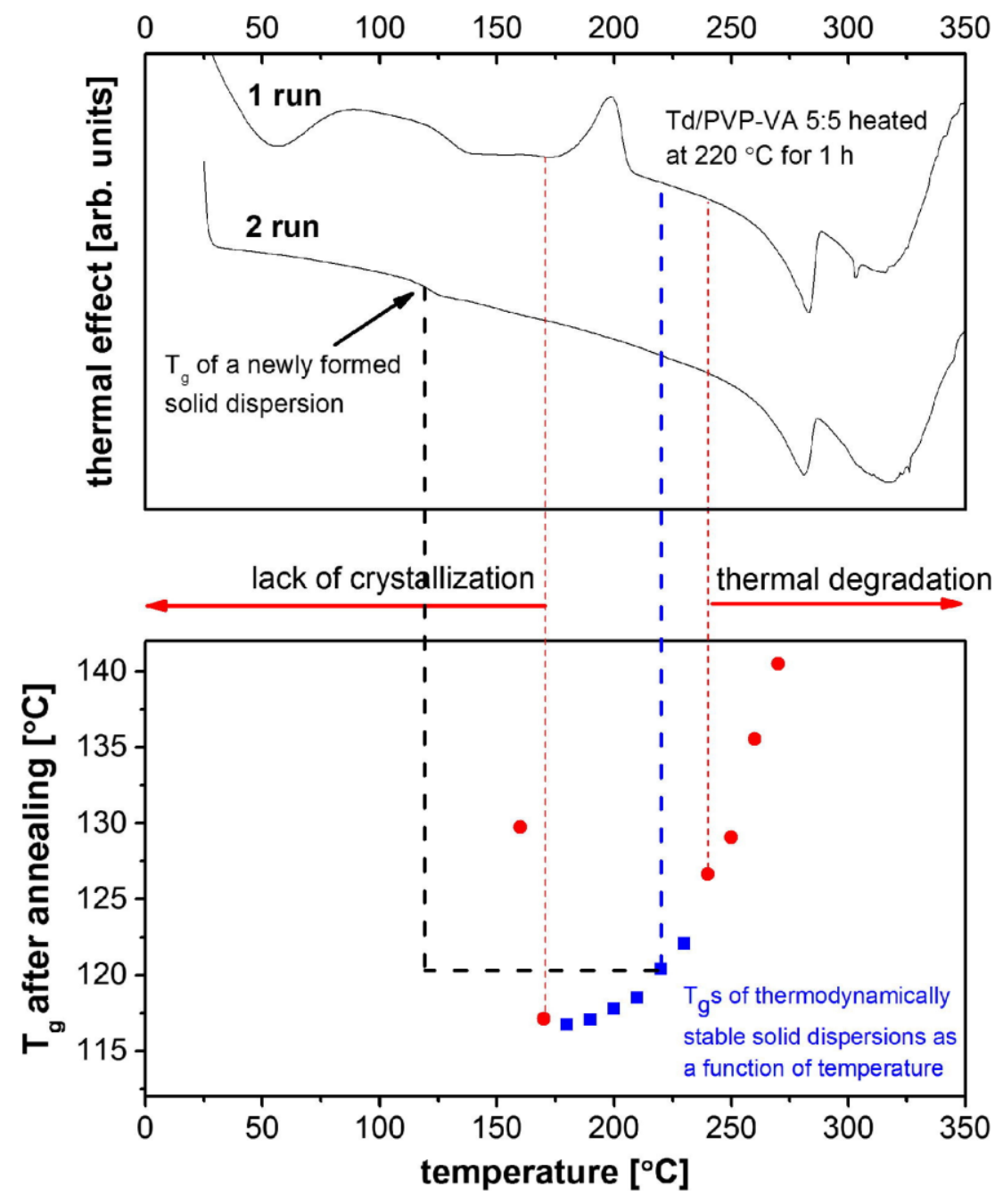


Fig. 6. The procedure for the determination of tadalafil (Td) thermodynamic solubility in PVPVA. The supersaturated 5:5 (w/w) Td/PVP-VA solid dispersion was annealed for one hour at $220{ }^{\circ} \mathrm{C}$ ( 1 run) to crystallize the excess of Td (top graph). The sample was subsequently cooled and heated (at $10{ }^{\circ} \mathrm{C} / \mathrm{min}$ ) to investigate its new $T_{g}$ ( 2 run), depicted as the blue square in the bottom graph. The new $T_{g}$ refers to the composition of a solid dispersion which is stable at $220{ }^{\circ} \mathrm{C}$ and therefore to the thermodynamic solubility of Td in PVP-VA at this temperature. By repeating this step at different annealing temperatures ranging from $180{ }^{\circ} \mathrm{C}$ to $230{ }^{\circ} \mathrm{C}$ it was possible to determine the relationship between $T_{g} \mathrm{~s}$ of stable solid dispersions and the temperature. Annealing at temperatures below $180{ }^{\circ} \mathrm{C}$ and above $230{ }^{\circ} \mathrm{C}$ yielded unreliable $T_{g} \mathrm{~S}$ (red dots) resulted from either the lack of crystallization or thermal degradation of components.

Td/PVP-VA solid dispersion $(5: 5, \mathrm{w} / \mathrm{w})$ was annealed for one hour at twelve different temperatures ranging from $160{ }^{\circ} \mathrm{C}$ to $270{ }^{\circ} \mathrm{C}$ but only the temperature range from 180 to $230{ }^{\circ} \mathrm{C}$ appeared to be suitable for analysis (Fig. 6). Annealing below this temperature range resulted in higher $T_{g} \mathrm{~s}$, what indicated the greater content of $\mathrm{Td}$ in the newly formed solid dispersions. As the solubility in polymer decreases with decreasing temperature this must have been the result of incomplete crystallization. Indeed, as shown in the thermogram of the Td/PVP-VA solid dispersion $(5: 5, \mathrm{w} / \mathrm{w})$ in Fig. 3b, temperatures of $160{ }^{\circ} \mathrm{C}$ and $170{ }^{\circ} \mathrm{C}$ are located below and at the beginning of Td crystallization, respectively. The solid dispersion annealed at $160{ }^{\circ} \mathrm{C}$ exhibits the glass transition at $129.73 \pm 0.24{ }^{\circ} \mathrm{C}$, which refers to $53 \%$ content of $\mathrm{Td}$ and indicates a lack of crystallization at this temperature.

Annealing of the Td/PVP-VA solid dispersion (5:5, w/w) at $260{ }^{\circ} \mathrm{C}$ and $270{ }^{\circ} \mathrm{C}$ resulted in $T_{g} \mathrm{~S}$ implying that the Td solubility significantly exceeds the nominal 50\% (Fig. 6). The possible reason of this could be aforementioned thermal degradation of components leading to unreliable results. Therefore, to validate this, crystalline Td and PVP-VA were separately subjected to the same annealing conditions using thermogravimetric analysis. The thermal decomposition of samples after one-hour annealing at different temperatures is shown as their percentage loss of weight in Table 1. The results present significant degradation of components above $240{ }^{\circ} \mathrm{C}$, which was additionally confirmed by the change of powder's color from white to brown. Therefore, $230^{\circ} \mathrm{C}$ was selected as the upper temperature limit as confirmed by acceptably low level of degradation of Td and PVP-VA.

Table 1. The percentage of weight loss (by TGA) of crystalline tadalafil and PVP-VA annealed for an hour at the different temperatures. 


\begin{tabular}{lllllr}
\hline \multicolumn{5}{c}{ The percentage weight loss of samples (\%) } \\
\cline { 2 - 6 } & $210^{\circ} \mathrm{C}$ & $230^{\circ} \mathrm{C}$ & $240^{\circ} \mathrm{C}$ & $250{ }^{\circ} \mathrm{C}$ & $270{ }^{\circ} \mathrm{C}$ \\
\hline Tadalafil & 1.05 & 1.11 & 1.09 & 1.72 & 7.08 \\
PVP-VA & 2.14 & 4.20 & 6.83 & 9.32 & 20.97 \\
\hline
\end{tabular}

In the protocol introduced by Mahieu and coworkers [12] $T_{g} \mathrm{~s}$ of solid dispersions were lower than that of the pure polymer, as a result of a low $T_{g}$ of the drug substance. It was highlighted that this plasticizing effect increases molecular mobility and thus accelerates the equilibration step when compared to the reverse process of drug dissolution in a polymer. In spite of the fact that the $T_{g} \mathrm{~s}$ of Td/PVP-VA solid dispersions are greater than that of the pure polymer, the process of crystallization occurs quite effectively. Crystallization of Td gradually decreases the $T_{g}$ of solid dispersion and therefore increases mobility of molecules, which may accelerate the equilibration step. In this work the one-hour annealing time appeared to be sufficient in comparison with the two-hour annealing step in Mahieu's protocol.

Annealing the Td/PVP-VA solid dispersion $(5: 5, \mathrm{w} / \mathrm{w})$ in the accepted range of temperatures (180 to $230{ }^{\circ} \mathrm{C}$ ) resulted in six solid dispersions differing in their $T_{g}$ s. Each $T_{g}$ referred to the composition of the solid dispersion, which was stable at the temperature of annealing. The content of Td, that is intrinsic solubility of Td in PVP-VA, was subsequently calculated based on the empirically obtained relationship between $T_{g}$ s and the composition of solid dispersions (Fig. 5). These values (black squares, Fig. 7) were fitted to the exponential function $\left(R^{2}=0.9891\right)$ according to the following model (Eq. (4)):

$$
m_{(\mathrm{Td})}=\mathrm{Ae}^{\mathrm{BT}}+\mathrm{C}
$$

where $m_{(\mathrm{Td})}$ is Td mass fraction in a solid dispersion, $T$ is a temperature expressed in $K$, while A, B, C adopted values of $8.75 \times 10^{-10}, 0.037$ and 0.205 , respectively (black line, Fig. 7). Since at lower temperatures the first part of the expression takes on low values the intercept (C) indicates calculated solubility at $25^{\circ} \mathrm{C}$, the most common storage temperature of solid oral dosage forms, which is $20.5 \%$ (Fig. 7). 


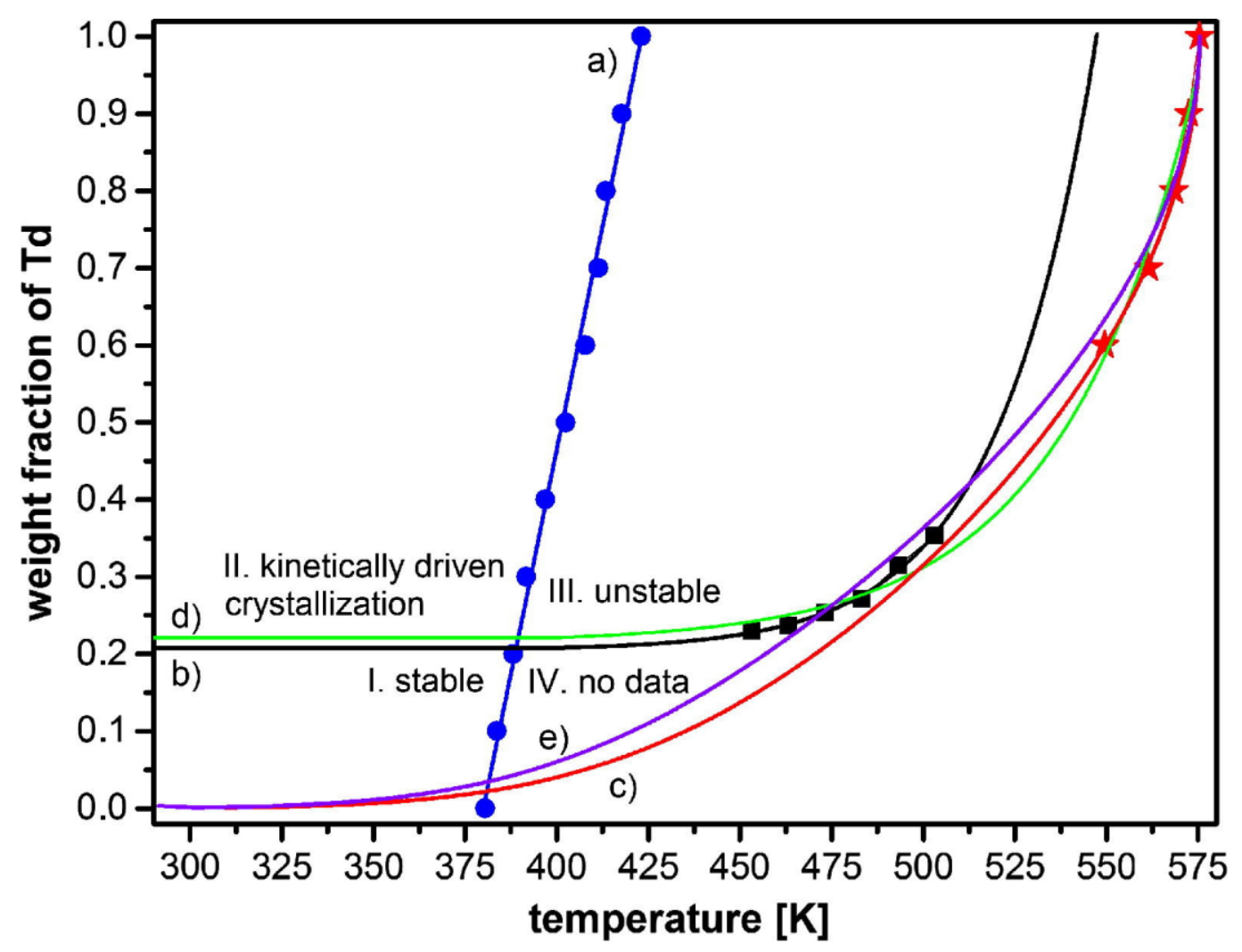

Fig. 7. The phase diagram of tadalafil (Td) and PVP-VA. The blue connected dots present the empirical relationship between Tgs and the composition of Td/PVP-VA solid dispersions (blue line, a). The black squares demonstrate thermodynamic solubility of Td in PVP/VA fitted to the exponential function and measured experimentally based on the Td crystallization from the supersaturated solid dispersion in the temperature range from 180 to $230{ }^{\circ} \mathrm{C}$ (black line, b) (Section 3.1.3). The red stars are experimental melting points of Td measured as described in Section 3.1.4 (HSDSC) fitted to the Flory-Huggins equation (red line, c). The green (d) and purple (e) lines represent curves based on both (black squares and red stars) sets of values fitted to the exponential and Flory-Huggins models, respectively.

In the broad range of 0 to $200{ }^{\circ} \mathrm{C}$ the Td solubility varies by no more than $5 \%$ but at higher temperatures it increases rapidly. At $180{ }^{\circ} \mathrm{C}$, where the crystallization of Td starts, the solubility is $22.8 \%$ which is reflected in thermograms of solid dispersions (Figs. $3 \mathrm{a}$ and $3 \mathrm{~b}$ ). The Td/PVPVA solid dispersion $(3: 7$, w/w) with $30 \%$ content of $\mathrm{Td}$ is the first for which a very broad thermal event of Td crystallization can be observed. Moreover, the phenomenon of subsequent dissolving finally finds support in the increased solubility of Td in PVP-VA in this range of temperatures. 


\subsubsection{Modified melting point depression - HSDSC}

While conventional methods employed to determine solubility of drugs in polymer typically use slow heating rates $[18,17]$ to attain solubility equilibrium as close as practically achievable, this is not always possible especially when the drug (or polymer) is known to thermally decompose. On the other hand, by applying fast heating rates decomposition upon/after melting can be diminished or eliminated [14]. A combination of a fast heating rate, to prevent sample decomposition, with an annealing step, as previously used by Sun et al. [17] and shown to obtain solubility results comparable with those yielded using only slow heating rates and no annealing, was applied and investigated as a novel approach to complement the method based on solid dispersions (Section 3.1.3).

The melting points of $\mathrm{Td}$ measured at $200{ }^{\circ} \mathrm{C} / \mathrm{min}$ are presented in Fig. 7 (star symbols). They were fitted to the Flory-Huggins equation (Eq. (5)):

$$
\left(\frac{1}{T_{m}}+\frac{1}{T_{o}^{m}}\right)=\frac{-R T}{\Delta H_{\text {fus }}}\left[\ln \phi+\left(1-\frac{1}{m}\right)(1-\phi)+\chi(1-\phi)^{2}\right]
$$

where $T_{m}$ and $T_{m}{ }^{0}$ are the melting points of Td in the binary mixture and pure drug respectively, $\Delta H_{\text {fus }}$ is the heat of fusion of pure crystalline $\mathrm{Td}, \phi$ is the volume fraction of $\mathrm{Td}, m$ is the volume ratio of PVP-VA to Td and $\chi$ is the Flory-Huggins interaction parameter.

The $\chi$ parameter was calculated to be -1.80 , which indicates good miscibility of components and is in agreement with the single $T_{g}$ determined for each of the solid dispersions (Fig. 3a). The solid-liquid line is presented in Fig. 7(red line) and while it well described the experimental points, the extrapolated drug solubility at $25{ }^{\circ} \mathrm{C}$ was estimated at only $\sim 0.1 \%$, in disagreement with the Td solubility of $\sim 20 \%$ as described in Section 3.1.3. This shows inadequacy of the Flory-Huggins model in relation to describing solubility of Td in PVP-VA as substantiated by stability studies (Section 3.2). A poor fit of this model was already reported by Sun et al. [17] for the mannitol/PVP system.

Surprisingly, as shown in Fig. 7, the points obtained by annealing solid dispersions (Section 3.1.3) followed the liquid-solid line based on the HSDSC measurements (this section), but each dataset covered a different $\mathrm{Td}$ weight fraction. This reveals that both methods are complementary and provide information on Td solubility encompassing a wide range of drug 
contents when considered collectively. Both sets of values yielded by the different approaches were fitted jointly to the exponential (Eq. (4)) and Flory-Huggins model (Eq. (5)), which resulted in green and purple curves, respectively, presented in Fig. 7.

\subsection{Accelerated aging tests}

The main goal of accelerated aging tests was to investigate physical stability of Td/PVP-VA amorphous solid dispersions with respect to the phase diagram depicted in Fig. 7. Thermodynamic properties resulting from the drug substance solubility in a polymer as well as kinetic properties arising from $T_{g}$ s of solid dispersions are two components that are crucial in estimating the physical stability of solid dispersions. The study was carried out on nine amorphous Td/PVP-VA solid dispersions and amorphous $\mathrm{Td}$ so that the impact of the supersaturation degree and the $T_{g}$ on the rate of crystallization could be assessed.

Neither amorphous Td nor none of the solid dispersions subjected to the aging conditions of $25{ }^{\circ} \mathrm{C}$ and $75 \% \mathrm{RH}$ as well as $40{ }^{\circ} \mathrm{C}$ and $0 \% \mathrm{RH}$ revealed crystallization of $\mathrm{Td}$ throughout the two-month study. However, the simultaneous increase in both temperature and humidity to $40{ }^{\circ} \mathrm{C}$ and $75 \% \mathrm{RH}$ led to the physical instability of samples, as confirmed by PXRD (Table 2). The fastest crystallization, already visible in the first day of storage, occurred in Td/PVPVA solid dispersions with the lowest content of Td. The crystallization rate decreased with the decreased amount of PVP-VA and was the lowest for Td without excipients. The reason for such behavior lies in high and low hygroscopicity of the polymer and hydrophobic $\mathrm{Td}$, respectively. Sorption of moisture by solid dispersions led to the reduction of their $T_{g} \mathrm{~s}$, what was particularly pronounced for 1:9-3:7 (w/w) Td/PVP-VA solid dispersions, and the morphology of the powders changed into the glassy appearance. Crystallization observed for the Td/PVP-VA solid dispersions (1:9 and 2:8, w/w), suggested as being undersaturated by the phase diagram as described in Section 3.1.3 (based on annealing of solid dispersions), at a temperature of $40{ }^{\circ} \mathrm{C}$ indicates poor applicability of phase diagrams when samples are stored in the presence of humidity. Calculated solubility of Td in PVP-VA refers to a two-component system which may be disturbed by the presence of a third component that is water molecules in this case.

Table 2. Crystallization behavior of amorphous tadalafil (Td) and 1:9-9:1 (w/w) Td/PVP-VA solid dispersions subjected to accelerated aging tests $\left(40{ }^{\circ} \mathrm{C}\right.$ and $75 \% \mathrm{RH}, 80{ }^{\circ} \mathrm{C}$ and $\left.0 \% \mathrm{RH}\right)$ analyzed after specified time intervals by PXRD. 


\begin{tabular}{lll}
\hline & \multicolumn{2}{l}{ Beginning of crystallization under storage conditions } \\
\cline { 2 - 3 } & $40{ }^{\circ} \mathrm{C}, 75 \% \mathrm{RH}$ & $80^{\circ} \mathrm{C}, 0 \% \mathrm{RH}$ \\
\hline 1 day & $1: 9-3: 7$ Td/PVP-VA & Amorphous Td 7:3-9:1 Td/PVP-VA \\
1 week & $4: 6-9: 1$ Td/PVP-VA & - \\
2 weeks & Amorphous Td & $3: 7-6: 4$ Td/PVP-VA \\
1 month & - & - \\
2 months & - & - \\
\hline
\end{tabular}

Therefore, the crystallization tendency with regard to Td thermodynamic solubility in PVPVA was investigated setting the humidity and temperature level to $0 \%$ and $80{ }^{\circ} \mathrm{C}$, respectively. In contrast to the previous study, the fastest crystallization observed after one day of storage occurred in amorphous Td and the solid dispersions with the lowest content of PVP-VA (Table 2). Physical instability among 3:7-6:4 (w/w) Td/PVP-VA solid dispersions was detected after two weeks (Fig. 8), while no crystallization occurred in solid dispersions (1:9 and 2:8, w/w) even after two months of storage (Fig. 9). This is a strong evidence that in the absence of moisture PVP-VA increases the physical stability of amorphous Td despite a reduction in $T_{g} \mathrm{~S}$ of binary systems. Moreover, this result suggests that the simple exponential model, but not the Flory-Huggins equation, was able to fit the experimental data better and give a more accurate estimation of Td solubility in PVP-VA. The physical stability of undersaturated solid dispersions, in respect of the drug content, is better, which is of a key importance when the planning solid dosage form formulations.

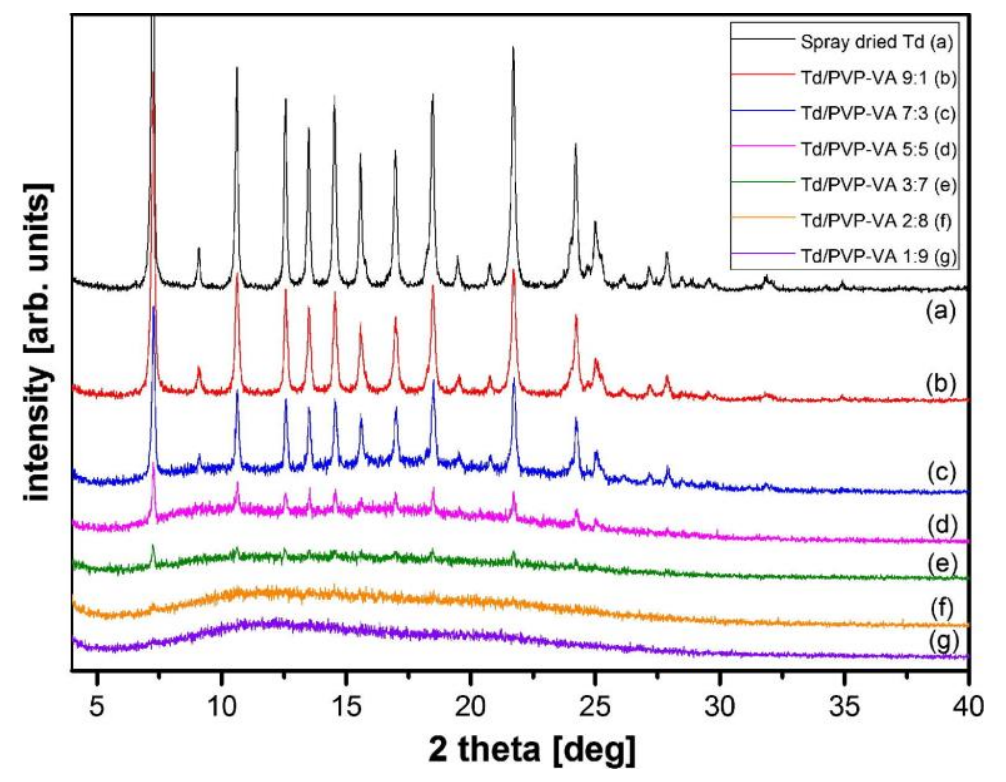

Fig. 8. X-ray powder diffraction patterns of spray dried tadalafil (Td) (a), 9:1-1:9 (w/w) $\mathrm{Td} / \mathrm{PVP}-\mathrm{VA}$ solid dispersions (b-g) obtained after 2 weeks of storage at $80{ }^{\circ} \mathrm{C}$ and $0 \% \mathrm{RH}$. 


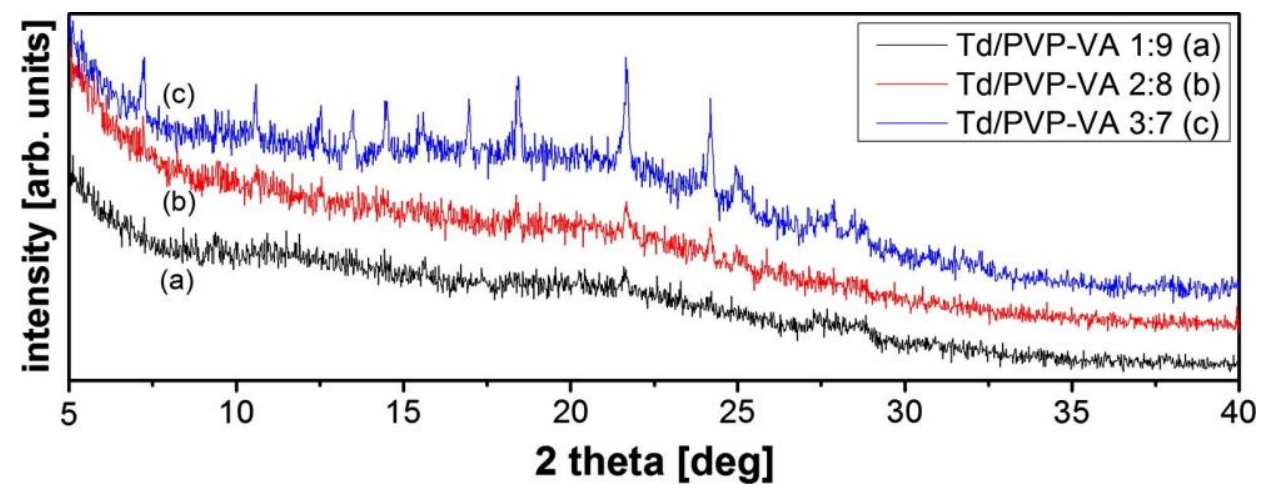

Fig. 9. X-ray powder diffraction patterns of 1:9 (w/w) Td/PVP-VA solid dispersion (a), 2:8 (w/w) Td/PVP-VA solid dispersion (b), 3:7 (w/w) Td/PVP-VA solid dispersion (c) obtained after 2 months of storage at $80{ }^{\circ} \mathrm{C}$ and $0 \% \mathrm{RH}$.

Lack of crystallization for the Td/PVP-VA solid dispersions (1:9-2:8, w/w) was additionally shown in a hot stage microscopy study, which validated the proposed exponential model. When heating the Td/PVP-VA solid dispersion $(3: 7, \mathrm{w} / \mathrm{w})$ at a $5{ }^{\circ} \mathrm{C} / \mathrm{min}$ rate crystals of $\mathrm{Td}$ appeared at $180{ }^{\circ} \mathrm{C}$ and crystallization was more pronounced in cooling the sample (Fig. 10a). This is in agreement with the crystallization events visible in DSC thermograms (Fig. 3b). On the contrary, no evidence of crystallization was provided while heating the 2:8 (w/w) Td/PVP-VA solid dispersion (Fig. 10b).
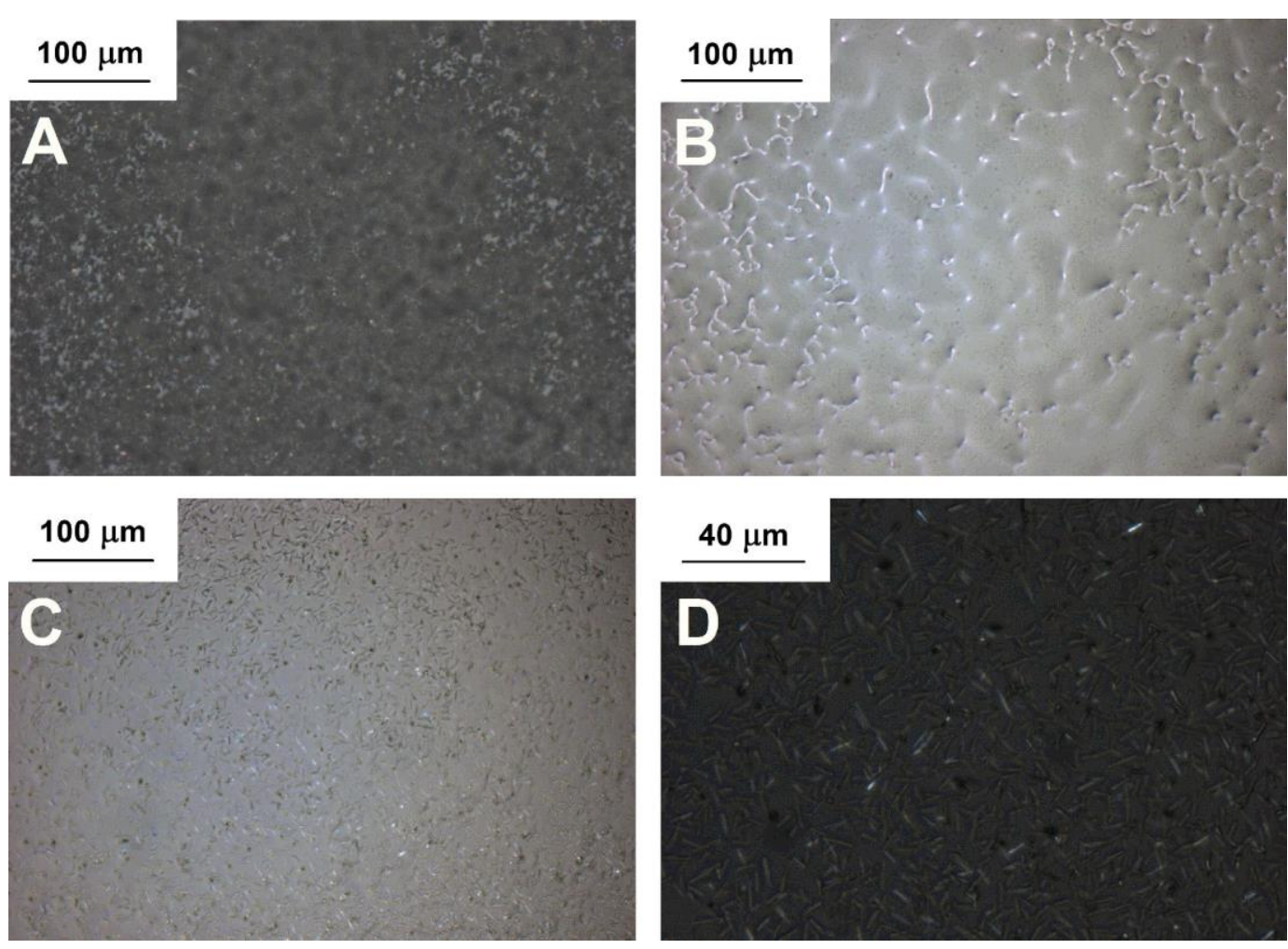
Fig. 10a. HS PLM images of a Td/PVP-VA $(3: 7, \mathrm{w} / \mathrm{w})$ solid dispersion (heating rate of $\left.5{ }^{\circ} \mathrm{C} / \mathrm{min}\right)$ at $25^{\circ} \mathrm{C}(\mathrm{A}), 180{ }^{\circ} \mathrm{C}(\mathrm{B}), 200{ }^{\circ} \mathrm{C}(\mathrm{C})$ and upon cooling (D).
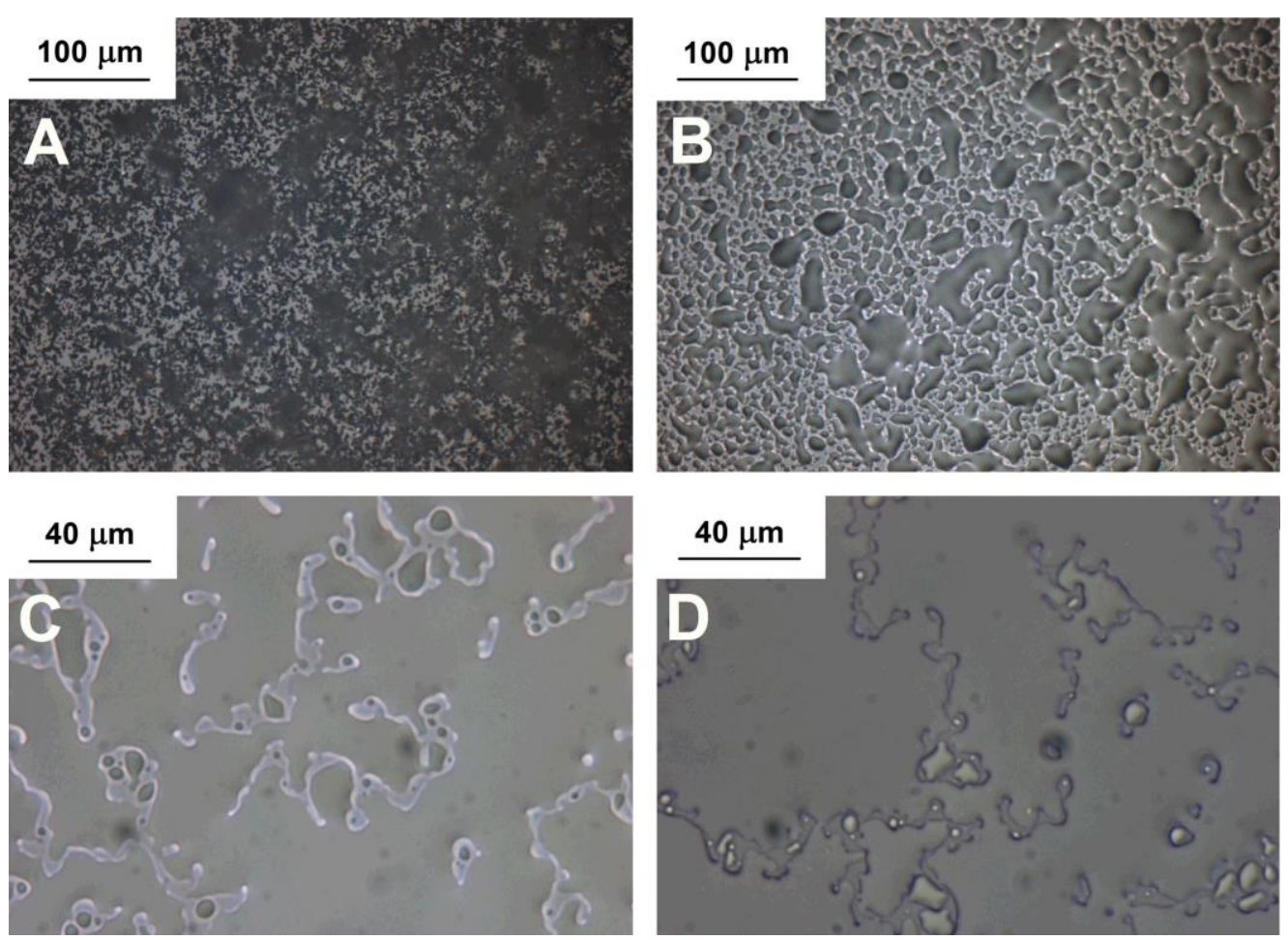

Fig. 10b. HS PLM images of a Td/PVP-VA $(2: 8$, w/w) solid dispersion (heating rate of $\left.5{ }^{\circ} \mathrm{C} / \mathrm{min}\right)$ at $25^{\circ} \mathrm{C}(\mathrm{A}), 180{ }^{\circ} \mathrm{C}(\mathrm{B}), 200{ }^{\circ} \mathrm{C}(\mathrm{C})$ and upon cooling (D).

To investigate the physical stability of undersaturated systems above their $T_{g}$ s (Fig. 7) Td/PVPVA solid dispersions (1:9 and 2:8, w/w) were also stored at $130{ }^{\circ} \mathrm{C}$ and $0 \% \mathrm{RH}$ for 2 weeks. However, an immediate color change, from white to orange, was noticed. The change in appearance may suggest decomposition of the samples; however, they remained amorphous for the duration of this study.

\section{Conclusions}

Nine Td/PVP-VA solid dispersions varied in terms of quantitative composition $(1: 9-9: 1, \mathrm{w} / \mathrm{w})$ were successfully obtained by spray-drying. Their amorphous nature, supersaturated character and molecular level of mixing (a solid solution structure) were subsequently confirmed using DSC, PXRD and calculation of Hansen total solubility parameters. Good miscibility and the lack of specific interactions between Td and PVP-VA were additionally reflected in the good agreement between the experimental and theoretical (based on the Gordon-Taylor equation) 
$T_{g}$ S of the solid dispersions. The linear relationship between $T_{g} \mathrm{~s}$ and the content of $\mathrm{Td}$ in binary systems enabled to introduce a unique protocol of the drug-polymer solubility determination.

This approach assumed crystallization of Td from a supersaturated solid dispersion and enabled the solubility estimations at lower temperatures than the drug melting point $\left(302.3{ }^{\circ} \mathrm{C}\right)$ to be conducted. It was important as at this melting temperature the Td and PVP-VA were seen to thermally decompose. Therefore, this work for the first time describes the calculation of the drug-polymer solubility as a function of temperature in the case of thermal degradation of both components. The obtained relationship was fitted to the exponential function, with the calculated solubility of $20.5 \%$ at $25{ }^{\circ} \mathrm{C}$.

The new, based on annealing of physical mixtures and applying a high heating rate, protocol for determining the melting point depression of $\mathrm{Td}$ was shown as complementary to the annealing of solid dispersion approach. Each of the methods produced a dataset covering a different range of Td concentration. The Flory-Huggins model fitted the experimental data poorly and Td solubility in PVP-VA was estimated by this equation at only around $0.1 \%$ at $25{ }^{\circ} \mathrm{C}$.

Td solid dispersions and amorphous $\mathrm{Td}$ subjected to the accelerated aging tests revealed physical instability under conditions of $40{ }^{\circ} \mathrm{C}$ and $75 \% \mathrm{RH}$ and $80{ }^{\circ} \mathrm{C}$ and $0 \% \mathrm{RH}$. In the presence of moisture, Td crystallization began in samples with the lowest content of the drug (10-30\%), which was the result of high hygroscopicity of the polymer, leading to the moisture sorption and lowering in $T_{g}$ s. However, the calculated thermodynamic solubility of Td (based on the exponential model) was in accordance with results of the stability test carried out at $80{ }^{\circ} \mathrm{C}$ and $0 \% \mathrm{RH}$, in which Td solid dispersions containing $10-20 \%$ of the drug were the only systems that did not crystallize within two months. The lack of Td crystallization in these systems was additionally confirmed by hot stage polarized light microscopy. This shows that despite the decreased $T_{g}$ s the physical stability of the non-supersaturated Td solid dispersions may be mainly affected by thermodynamics.

\section{Acknowledgments}

This project was supported by the Ministry of Science and Higher Education Republic of Poland, from the quality-promoting subsidy, under the Leading National Research Centre (KNOW) program for the years 2012-2017, by grant awarded by National Centre for Science 
(NCN) - "OPUS" Nr DEC-2012/05/B/N27/03233 and by the Synthesis and Solid State Pharmaceutical Centre funded by Science Foundation Ireland under grant number 12/RC/2275. The authors would like to thank Ms. Naila Mugheirbi from the School of Pharmacy and Pharmaceutical Sciences, Trinity College Dublin for her SEM analysis.

\section{References}

[1] Y. Amharar, V. Curtin, K.H. Gallagher, A.M. Healy, Solubility of crystalline organic compounds in high and low molecular weight amorphous matrices above and below the glass transition by zero enthalpy extrapolation, Int.J. Pharm. 472 (2014) 241-247.

[2] J.A. Baird, B. Van Eerdenbrugh, L.S. Taylor, A classification system to assess the crystallization tendency of organic molecules from undercooled melts,J. Pharm. Sci. 99 (2010) 3787-3806.

[3] C. Brough, R.O. Williams III, Amorphous solid dispersions and nano-crystal technologies for poorly water-soluble drug delivery, Int. J. Pharm. 453 (2013) 157-166.

[4] A.K. Chaturvedi, A. Verma, Solubility enhancement of poorly water soluble drugs by solid dispersion, Eur. J. Pharm. Sci. 3 (2012) 26-34.

[5] L. Di, P.V. Fish, T. Mano, Bridging solubility between drug discovery and development, Drug Discovery Today 17 (2012) 486-495.

[6] D. Greenhalgh, A. Williams, P. Timmins, P. York, Solubility parameters aspredictors of miscibility in solid dispersions, J. Pharm. Sci. 88 (1999) 1182-1190.

[7] M. Gordon, J.S. Taylor, Ideal copolymers and the second-order transitions of synthetic rubbers. 1. Non-crystalline copolymers, J. Appl. Chem. 2 (1952) 493-500.

[8] H. Grohganz, P.A. Priemel, K. Löbmann, L.H. Nielsen, R. Laitinen, A. Mullertz, G. Van Den Mooter, T. Rades, Refining stability and dissolution rate of amorphous drug formulations, Expert Opin. Drug Deliv. 11 (2014) 977-989.

[9] E. Karavas, G. Ktistis, A. Xenakis, E. Georgarakis, Miscibility behavior and formation mechanism of stabilized felodipine-polyvinylpyrrolidone amorphous solid dispersions, Drug Dev. Ind. Pharm. 31 (2005) 473-489. 
[10] H. Konno, L.S. Taylor, Ability of different polymers to inhibit the crystallization of amorphous felodipine in the presence of moisture, Pharm. Res. 25 (2008) 969-978.

[11] C. Leuner, J. Dressman, Improving drug solubility for oral delivery using solid dispersions, Eur. J. Pharm. Biopharm. 50 (2000) 47-60.

[12] A. Mahieu, J.-F. Willart, E. Dudognon, F. Daneìde, M. Descamps, A new protocol to determine the solubility of drugs into polymer matrixes, Mol. Pharm. 10 (2013) 560-566.

[13] P.J. Marsac, S.L. Shamblin, L.S. Taylor, Theoretical and practical approaches for prediction of drug-polymer miscibility and solubility, Pharm. Res. 23 (2006) 2417-2426.

[14] K.J. Paluch, T. McCabe, H. Müller-Bunz, O.I. Corrigan, A.M. Healy, L. Tajber, Formation and physicochemical properties of crystalline and amorphous salts with different stoichiometries formed between ciprofloxacin and succinic acid, Mol. Pharm. 10 (2013) 36403654.

[15] S. Qi, P. Belton, K. Nollenberger, N. Clayden, M. Reading, D.Q.M. Craig, Characterisation and prediction of phase separation in hot-melt extruded solid dispersions: a thermal, microscopic and NMR relaxometry study, Pharm. Res. 27 (2010) 1869-1883.

[16] R. Simha, R.F. Boyer, On a general relation involving the glass temperature and coefficients of expansion of polymers, J. Chem. Phys. 37 (1962) 1003-1007.

[17] Y. Sun, J. Tao, G.G.Z. Zhang, L. Yu, Solubilities of crystalline drugs in polymers: An improved analytical method and comparison of solubilities of indomethacin and nifedipine in PVP, PVP/VA, and PVAc, J. Pharm. Sci. 99 (2010) 4023-4031.

[18] J. Tao, Y. Sun, G.G.Z. Zhang, L. Yu, Solubility of small-molecule crystals in polymers: d-Mannitol in PVP, indomethacin in PVP/VA, and nifedipine in PVP/ VA, Pharm. Res. 26 (2008) 855-864.

[19] D.W. van Krevelen, P.J. Hoftyzer, Properties of Polymers, their Estimation and Correlation with Chemical Structure, second ed., Elsevier, Amsterdam, 1976.

[20] C.L.-N. Vo, C. Park, B.-J. Lee, Current trends and future perspectives of solid dispersions containing poorly water-soluble drugs, Eur. J. Pharm. Biopharm. 85 (2013) 799-813. 
[21] H.D. Williams, N.L. Trevaskis, S.A. Charman, R.M. Shanker, W.N. Charman, C.W. Pouton, C.J.H. Porter, Strategies to address low drug solubility in discovery and development, Pharmacol. Rev. 65 (2013) 315-499.

[22] K. Wlodarski, W. Sawicki, K.J. Paluch, L. Tajber, M. Grembecka, L. Hawelek, Z. Wojnarowska, K. Grzybowska, E. Talik, M. Paluch, The influence of amorphization methods on the apparent solubility and dissolution rate of tadalafil, Eur. J. Pharm. Sci. 62 (2014) 132140.

[23] K. Wlodarski, W. Sawicki, K. Haber, J. Knapik, Z. Wojnarowska, M. Paluch, P. Lepek, L. Hawelek, L. Tajber, Physicochemical properties of tadalafil solid dispersions - Impact of polymer on the apparent solubility and dissolution rate of tadalafil, Eur. J. Pharm. Biopharm. 94 (2015) 106-115. 